\title{
Modelos e simulações em educação ambiental: Relato de experiências em sala de aula
}
Fábio Ferrentini Sampaio', Alexandre Neves Louzada ${ }^{2,4}$, Rodolfo Paolucci ${ }^{3,4}$, Rodrigo Leão de Moura, André Vidal ${ }^{4}$

\author{
${ }^{1}$ Programa de Pós-Graduação em Informática/UFRJ \\ ${ }^{2}$ Unigranrio - Universidade do Grande Rio - RJ - Brasil \\ ${ }^{3}$ Instituto de Comunicação e Informação Científica e Tecnológica em Saúde/FIOCRUZ \\ ${ }^{4}$ Fundação de Apoio à Escola Técnica do Estado do Rio de Janeiro - RJ - Brasil \\ ffs@nce.ufrj.br, professorlouzadalglobo.com, rpprjbregmail.com
}

\begin{abstract}
This paper presents a study on the implementation of educational activities using computational modeling for environmental education within the Project Model2Learn. The activities are part of the curriculum of a discipline in Computer Science in a State Technical School in Rio de Janeiro. Preliminary results of the study point to a commitment and interest of the students on the topics addressed.
\end{abstract}

Resumo. Este artigo apresenta um estudo sobre a aplicação de atividades pedagógicas utilizando modelagem computacional para a educação ambiental no âmbito do Projeto Model2Learn. As atividades fazem parte do currículo da disciplina de Informática de uma Escola Técnica Estadual no Rio de Janeiro. Os resultados preliminares do estudo apontam para um engajamento e interesse dos alunos sobre os temas tratados.

\section{Introdução}

O presente trabalho trata da aplicação pedagógica de modelos e simulações em educação ambiental, no âmbito do Projeto Model2Learn [Sampaio, 2011].

O projeto Model2Learn (M2L) visa a criação, na web, de uma comunidade virtual para compartilhamento de modelos, simulações, experiências pedagógicas e recursos multimídia sobre educação ambiental. O público alvo é composto, preferencialmente, de professores e alunos de ensino médio e superior.

As atividades envolvendo modelagem e simulação utilizam o ambiente computacional NETLOGO [Wilensky, 1999] e uma abordagem pedagógica em três níveis de interação definidos por Taylor e Sampaio (2010).

O projeto vem sendo desenvolvido com a participação de pesquisadores da UFRJ, professores e alunos de uma Escola Técnica Estadual do $2^{\circ}$. Segmento na cidade do Rio de Janeiro, apoiados pela FAPERJ.

Na seção 2 é apresentada a justificativa e relevância do Projeto, seguida pela seção 3, onde a estratégia pedagógica adotada no mesmo é tratada. A seção 4 apresenta os

1 Projeto financiado pela FAPERJ: E - 26/110.141/2012 
modelos e atividades preparadas para utilização no ano acadêmico de 2013 na Escola Técnica em questão. A seção 5 apresenta alguns resultados parciais, seguida das conclusões e trabalhos futuros (seção 6).

\section{Justificativas e Relevância}

A educação ambiental tornou-se obrigatória a partir da Lei 9.795 promulgada em 1999. No seu Art. $2^{\circ}$ afirma: "A educação ambiental é um componente essencial e permanente da educação nacional, devendo estar presente, de forma articulada, em todos os níveis e modalidades do processo educativo, em caráter formal e não-formal.".

Apesar de podermos afirmar que, de uma forma geral, o tema vem sendo tratado preferencialmente nas disciplinas de Geografia e Biologia, é possível hoje reconhecer a existência de abordagens interdisciplinares onde a ênfase não se dá apenas na apresentação de conceitos e idéias prontas.

Desta forma, o projeto M2L pode ser visto como uma possibilidade de instrumentalizar professores e alunos na construção de novas formas de ensinar e aprender, envolvendo estes últimos em atividades que possam ajudá-los a desenvolver habilidades e o senso crítico sobre questões relacionadas ao meio-ambiente (propor e testar hipóteses, capacidade de sintese e abstração, avaliar, interpretar e adaptar situações e problemas, entre outros) e suas inter-relações com outras áreas do conhecimento [Kahn et. al, 2010].

\section{Abordagem pedagógica}

A proposta pedagógica do Model2Learn (Figura 1) descreve três níveis de interação interdependentes envolvendo professores, alunos e modelos para o desenvolvimento de habilidades para modelagem computacional e para a promoção da aprendizagem sobre um determinado problema, dentro do contexto de educação ambiental [Taylor e Sampaio, 2010]. Estes níveis norteiam as atividades a serem trabalhadas em sala de aula a partir de uma abordagem construcionista [Papert, 1991].

No primeiro nível sugere-se a execução de uma versão inicial do modelo, propondo algumas questões simples e provocativas para familiarização com uma situação problema e as partes que a compõem.

No segundo nível de interação é apresentado um conjunto de questões mais complexas de tal forma que, para respondê-las, os alunos precisam alterar alguns parâmetros e valores iniciais, fazendo várias simulações do modelo proposto.

O terceiro e mais avançado nível propõe, através de perguntas e questionamentos, a alteração do próprio modelo para adaptá-lo a situações análogas, ou evoluí-lo para representar situações mais complexas. 


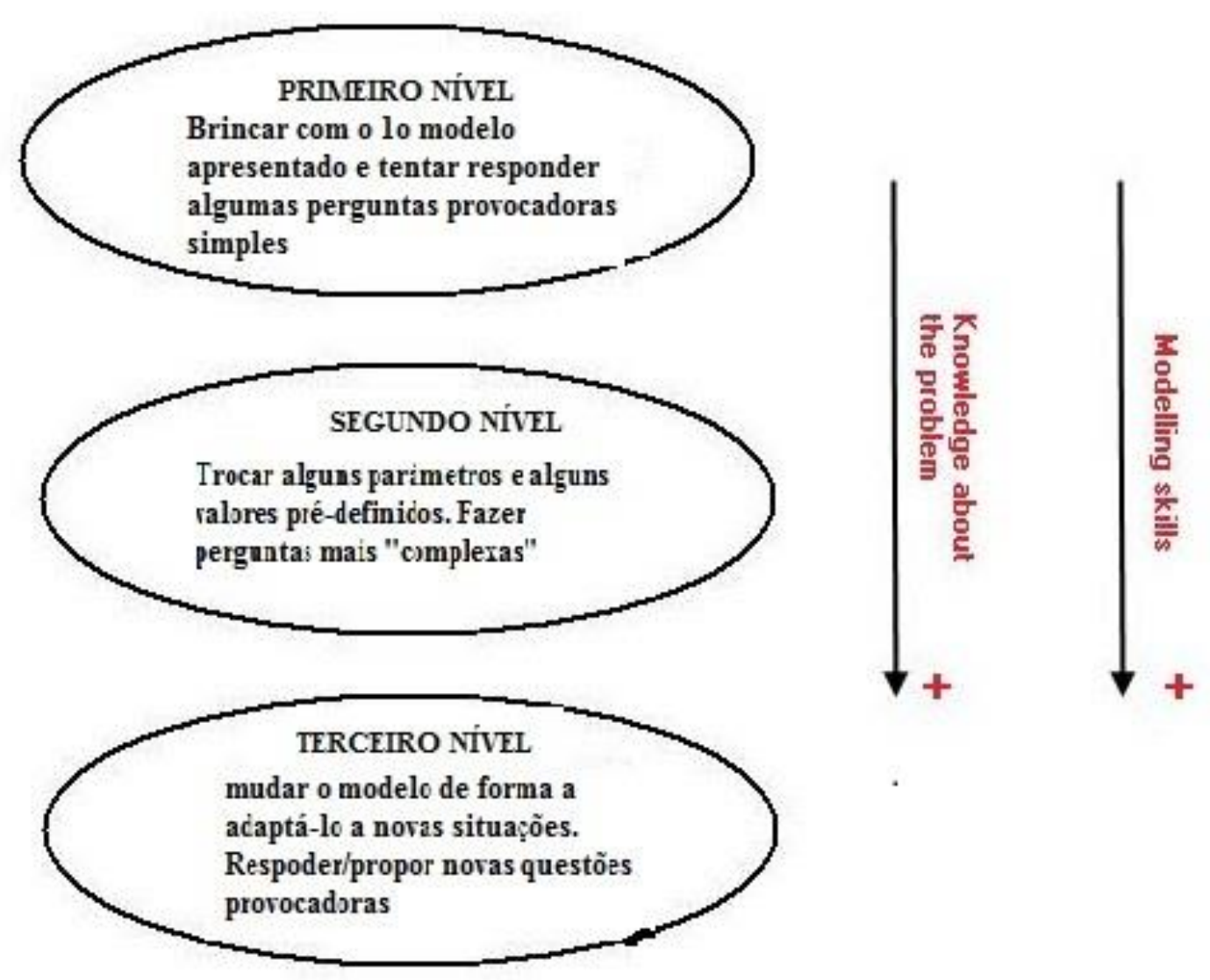

Figura 1: Proposta pedagógica do Model2Learn

\section{Modelos e Atividades Pedagógicas Desenvolvidas}

O trabalho em desenvolvimento na Escola Técnica iniciou em Abril de 2013 com a intenção de ocupar cerca de 11 aulas de 100 minutos ao longo do ano acadêmico. Cada atividade utiliza pelo menos duas aulas com um intervalo de uma ou duas semanas entre elas.

As aulas ocorrem dentro da grade curricular normal da Escola, ocupando tempos de uma disciplina de iniciação a programação de computadores.

Foram programados cinco temas, cada um deles tratando de um assunto diferente em Educação Ambiental e com alguma associação com o currículo das disciplinas do curso: Consumo de carne X Consumo de Cereais X Desmatamento; Queimadas; Ambientes coralíneos; Teoria de Gaia (Mundo das margaridas); Efeito Estufa.

Cada tema segue um roteiro pré-estabelecido com o seguinte formato geral:

$1^{\mathrm{o}}$. dia

- Motivação (uso de vídeos, perguntas provocativas, etc.);

- Apresentação de idéias e conceitos pertinentes ao tema em questão;

- Atividades em sala para ilustrar os conceitos e idéias apresentas (sem modelagem); 
- Proposta de atividades de continuidade e aprofundamento de questões no Facebook (realizadas ao longo da semana).

$2^{\circ}$. Dia

- Atividades no laboratório de informática explorando um modelo computacional sobre o tema em questão, implementado pela equipe do projeto.

A Escola Técnica Estadual onde o estudo está sendo realizado encontra-se em uma região na Zona Oeste da cidade do Rio de Janeiro, com moradores de classe média / média-baixa. A turma tem cerca de 22 alunos, composta majoritariamente por meninos. A idade varia entre 13 e 16 anos.

Na seção a seguir a atividade sobre a Teoria de Gaia - proposto por Lovelock (2006) - e o modelo "Mundo das Margaridas" são apresentados de forma mais detalhada.

\subsection{Atividade sobre a Teoria de Gaia e o Mundo das Margaridas}

A Teoria de Gaia de James Lovelock é uma evolução de sua hipótese postulada no início da década de 1970 de que a vida em nosso planeta mantém, ativamente, as condições da superfície sempre favoráveis aos organismos que a ocupam. Baseando-se em observações e modelos teóricos, ela descreve a Terra como um sistema retroalimentado que equilibra suas condições para garantir a sobrevivência das espécies [Lovelock, 2006].

Um destes modelos que ficou popularmente conhecido e elucida a teoria é o Mundo das Margaridas (Daisyworld em Inglês). O modelo foi implementado por Watson e Lovelock (1983) e descreve a interação de diferentes espécies de margaridas com o planeta e vice-versa. O modelo é uma simulação do planeta Terra com apenas duas espécies de plantas, margaridas brancas e pretas, competindo por recursos naturais.

Segundo a teoria, nos primórdios da vida solar, o planeta do modelo estaria mais frio, propiciando a vida das margaridas pretas. Com o passar dos anos e o aquecimento da Terra pelo Sol, as margaridas brancas encontram melhores condições de reprodução, fazendo com que a competição entre as espécies mantenha a temperatura próxima da ideal para a vida [Lovelock, 2006].

\subsubsection{O Modelo o Mundo das Margaridas Utilizado no Projeto}

O modelo Mundo das Margaridas presente na biblioteca do NetLogo [Novak \& Wilensky, 2006] e adaptado para o projeto M2L, tem como objetivo permitir aos seus usuários explorar algumas idéias sobre a Teoria de Gaia, trabalhando com um mundo hipotético semelhante ao nosso planeta, onde apenas duas espécies de margaridas coexistem: as claras e as escuras. Ambas competem por espaço na natureza e, dependendo das condições climáticas - aqui representadas apenas pela energia solar e o albedo das plantas e do solo - uma delas estará com melhores condições de sobreviver e de polinizar. No modelo, as margaridas pretas absorvem a luz solar, esquentando a si mesmas e ao planeta. As brancas, por sua vez, refletem a luz solar, arrefecendo o ambiente.

As margaridas e o solo possuem um albedo, representado como a porcentagem da energia na forma de calor que absorvem através da luz do sol. Considera-se o valor 1.0 para o parâmetro da luz solar como sendo a luminosidade média do nosso sol. Cada localização (patch) no ambiente de simulação do NETLOGO representa um espaço que 
pode estar vazio ou ocupado por um dos dois tipos de margaridas. A Figura 2 ilustra a visão do modelo preparado para ser executado.
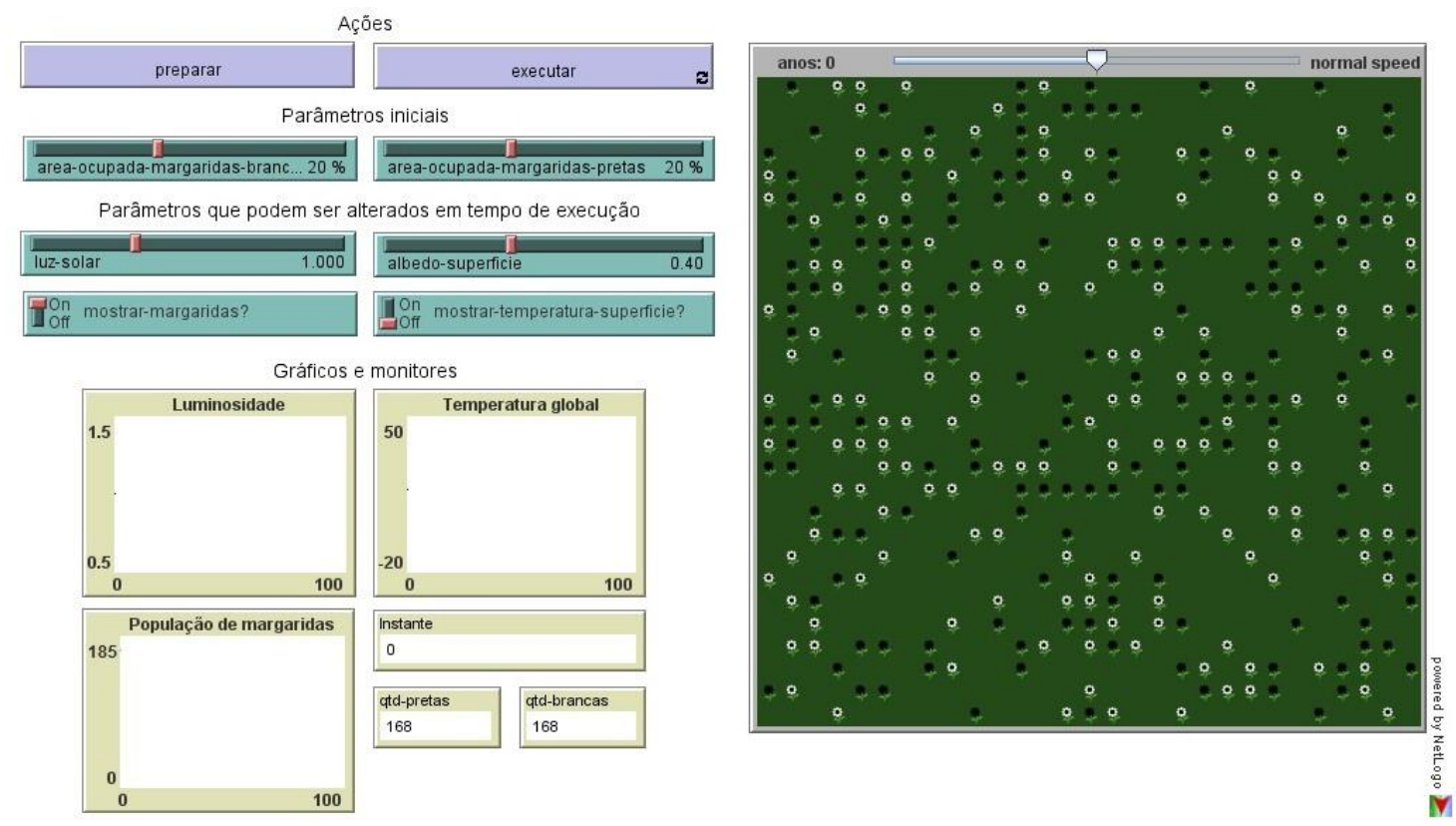

Figura 2. Modelo Daisyworld na versão Model2Learn

A cada instante da simulação alguns cálculos são efetuados de forma automática, atualizando os valores de temperatura nos patches, da margarida existente num determinado patch e da difusão de $50 \%$ da temperatura de um determinado patch para os seus vizinhos. Em seguida, é calculada a probabilidade de brotar uma nova margarida num patch vazio e vizinho a uma margarida existente. Essa probabilidade é dada por uma função parabólica que depende da temperatura local do patch ( o valor máximo da probabilidade ocorre a uma temperatura de $22,5^{\circ} \mathrm{C}$ ). $\mathrm{O}$ modelo também considera a idade máxima que uma margarida pode atingir para morrer, esvaziando o patch ocupado por ela a cada 25 interações.

O ambiente de simulação ainda apresenta gráficos para o acompanhamento da luz solar, temperatura global do ambiente (média das temperaturas dos patches) e das populações de margaridas.

Os usuários podem manipular alguns parâmetros: área inicial ocupada pelos dois tipos de margaridas, quantidade de luz solar incidente e albedo da superfície.

\subsubsection{O Roteiro de Trabalho dos Alunos}

Após uma apresentação inicial do modelo, os alunos, organizados em duplas, devem responder a um conjunto de perguntas apresentado em um questionário on-line. As perguntas aparecem na forma sequencial, respeitando o nível de complexidade detalhado na Figura 1. No entanto, as duplas têm a liberdade de respondê-las na ordem em que desejarem.

No primeiro nível de interação, questões simples são apresentadas: O que acontece com as populações de margaridas para os parâmetros iniciais ?; Os valores finais das 
populações são os mesmos após diferentes simulações ?; se iniciarmos a simulação com maior quantidade de um tipo de margaridas faz diferença ?; Caso a luz solar seja alterada para populações iniciais diferentes, o resultado é o mesmo? Por quê?

No segundo nível, para se obter as respostas às perguntas formuladas é enfatizada a modificação dos parâmetros e a interpretação dos resultados apresentados nos gráficos gerados pelas simulações realizadas pelas duplas: Quais situações provocam altas e baixas nas diferentes populações de margaridas ?; As margaridas influenciam na temperatura e vice-versa ?; $\mathrm{O}$ que acontece se as populações ficarem instáveis, com altas e baixas frequentes ?; Em que situações vemos a extinção de todas as espécies de margaridas? Pede-se justificativa.

Uma vez que os alunos envolvidos no trabalho ainda não possuem conhecimentos de programação NETLOGO, as perguntas de terceiro nível foram selecionadas com o intuito de fazer com que eles reflitam sobre possíveis novas situações análogas àquela trabalhada na atividade, sem no entanto terem que reprogramar o código fonte do modelo: O que acontece com o modelo se modificarmos o valor de energia (calor) transferido para os patches vizinhos? O que isso representa na situação real ?; Que outros exemplos de situações existentes na natureza têm comportamento semelhante à este representado no modelo ?.

\section{Resultados Parciais}

As atividades em sala de aula têm sido observadas pelos professores e pesquisadores participantes do Projeto que, após os encontros, produzem relatórios sobre as suas observações. Os relatórios são então consolidados num documento único que vem servindo para fazer adaptações nas tarefas ainda não realizadas. A seguir apresentamos as principais observações anotadas.

A turma tem iniciado as atividades de forma relativamente dispersa e, com o passar do tempo, vai se concentrando mais na fala do professor e/ou nas atividades propostas. A parir de perguntas e questionamentos apresentados, os alunos vão se engajando, preocupados em opinar, criticar e se fazer ouvir.

No segundo dia de atividade relativa a um determinado tema, tem-se percebido um amadurecimento no discurso por parte dos alunos. É possível que as atividades propostas no Facebook, e que devem ser realizadas por eles ao longo da semana, estejam também contribuindo para essa "maior desenvoltura".

Ainda sobre o Facebook, vale ressaltar que a atividade tem-se configurado com o perfil comumente encontrado em atividades à distância: postagens aleatórias e pico de discussão na véspera da data limite de finalização da tarefa.

A estratégia pedagógica de modelagem e simulação tem sido bem recebida, com as duplas procurando explorar os modelos a partir das perguntas propostas no questionário de atividades.

Percebe-se também que algumas duplas procuram explorar um pouco o código NetLogo, apesar de tal ação não ser obrigatória, nem - a principio - ajudar a resolver as tarefas propostas.

Quanto às estratégias de modelagem e simulação empregadas para resolver uma determinada questão, foi possível perceber uma certa ingenuidade por parte dos alunos. Em algumas situações executavam o modelo apenas uma vez para tentar verificar 
alguma mudança numa certa característica de comportamento do mesmo. Outras vezes executavam simulações procurando controlar certas variáveis do modelo, mas apesar de saberem escolhê-las corretamente, o set-up do período de tempo das simulações era insuficiente para perceber mudanças significativas nas variáveis estudadas.

Aqui, algumas hipóteses podem ser levantadas para justificar tal comportamento por parte dos alunos:

- É muito provável que este trabalho seja uma primeira oportunidade dada aos alunos (em ambiente escolar) de propor e testar hipóteses trabalhando em um cenário de "problemas abertos" [Ogborn, 1992].

- Os problemas tratados nas atividades, no mundo real, ocupam dezenas ou centenas de anos para evoluir. Valores estes que, para alunos adolescentes, ainda estão fora da sua experiência diária. Assim, é possível pensarmos que para eles prepararem o set-up de uma simulação utilizando um período de tempo de apenas 2 ou 3 anos, seja algo mais do que razoável.

Para as duas hipóteses apontadas, pode-se esperar que a experiência acumulada pela turma na resolução das atividades e problemas que estão por vir deverá contribuir para que o grupo apresente estratégias mais apropriadas (e sofisticadas) para solucionar as questões apresentadas.

\section{Conclusões e trabalhos futuros}

O presente trabalho trata da apresentação e discussão de um dos objetivos do projeto Model2Learn, qual seja, da implementação em sala de aula de atividades pedagógicas envolvendo modelagem computacional sobre temas em educação ambiental.

O projeto está em fase final de execução e os resultados encontrados têm sido satisfatórios.

Ao final do Projeto, o material desenvolvido (modelos e atividades), bem como os relatórios produzidos serão disponibilizados no portal e blog do Projeto (http://modeltolearn.blogspot.com.br/) a fim de que possam ser utilizados e aperfeiçoados por outros professores e pesquisadores.

\section{Referências}

Khan, K. ; Noble H. ; SAMPAIO, F. F. ; Hjorth A. (2010). Modelling4All: Ambiente na web 2.0 para a construção, simulação e compartilhamento de modelos baseados em agentes. In: Anais do XXI Simposio Brasileiro de Informática na Educação. João Pessoa.

Lovelock, J.E. (2006) A Vingança de Gaia. Editora Intrínseca, Rio de Janeiro.

Novak, M. and Wilensky, U. (2006). NetLogo Daisyworld model. http://ccl.northwestern.edu/netlogo/models/Daisyworld. Center for Connected Learning and Computer-Based Modeling, Northwestern University, Evanston, IL.

Ogborn, J. M. (1992). Modelling with the computer at all ages. Portugal Physics. 21(3/4), 99-114.

Papert, S. M. (1991) "Situating Constructionism". In: Constructionism, Edited by I. Harel and S. Papert. Norwood, NJ: Ablex Publishing. 
Sampaio, F. F. (2011). Projeto Model2Learn: Um portal na web 2.0 para ensinoaprendizagem de modelagem dinâmica e educação ambiental. http://www.nce.ufrj.br/GINAPE/publicacoes/Projetos/Proj_Model2Learn.pdf

Taylor, R., Sampaio, F. F. and Santos, R. M. (2010) "Model2Learn: An educational web-based tool to explore ideas about modelling and environment". In: XXI Simpósio Brasileiro de Informática na Educação. Anais do XXI Simpósio Brasileiro de Informática na Educação, João Pessoa.

Watson, A.J. and Lovelock, J.E. (1983) "Biological homeostasis of the global environment: the parable of Daisyworld", Tellus 35B, 286-289.

Wilensky, U. (1999). NetLogo. http://ccl.northwestern.edu/netlogo/. Center for Connected Learning and Computer-Based Modeling, Northwestern University, Evanston, IL. 